\title{
Psychosocial aspects in children and addescents with type I dm
}

\section{Introduction}

a. Type 1 diabetes is a chronic illness that is usually diagnosed in children, addescents (below the age of 20 years) and young adults. It is well established it is due to absence of insulin secretion.

b. In addition to daily injections, it involves many other lifestyle adjustments such as timing and nature of food consumption, regular exercise and blood glucose monitoring, especially in school of college going children.

c. Failure to follow any of these could lead to serious consequences in the form of hypoglycemia, ketoacidosis or micro and macro vascular complications may lead to severe immediate complication Liked KA, Hypo.

d. The International society for paediatric and Adolescent Diabetes (ISPAD) guidelines stated that "Psychosocial factors are the most important influences affecting the care and management of diabetes" and made the following three general recommendations.

\section{Psychiatric and eating disorders}

A. Managing Type 1 diabetes is particularly difficulty during adolescence.

Adolescence is a period of major physiological and psychological changes, and is also characterized by

\section{i. Striving for autonomy}

ii. Independence as against the restrictions imposed by the parents.

iii. Need for peer affiliation and acceptance

iv. Pre occupation with body.

All these clash with the diabetes regimen. Psychiatric and eating disorders are more frequent among adolescents with diabetes than among their non-diabetic peers, as well as substance abuse.

\section{Psychiatric and behavioural disorders}

A. Psychiatric and behavioural disorders in adolescents with TIDM are commonly seen and are categorized as either "Internalizing" - depression and anxiety or "Externalizing" - impulsivity, hyperactivity, aggression disorders.

B. In the initial period after diabetes diagnosis, children show difficulties in coping with the disease often presenting with feelings of sadness, withdrawal and anxiety.

C. Over $1 / 3$ rd of children with diabetes develop a psychiatric disorder within the first decade of disease onset, with depression, anxiety and behavioural disorders being the most common diagnoses.

i. Predisposing factors contributing to depression or anxiety disorders are:
a. Female gender
b. Poor glycemic control

Volume 4 Issue I - 2017

\author{
Jayalakshmi T, Munichoodappa C \\ Department of Diabetology, India
}

Correspondence: Munichoodappa, Department of Diabetology, The Bangalore Hospital, 202 RV Road, Bengaluru 560004, India, Email dr.munichoodappa@gmail.com

Received: January 17, 2017 | Published: January 24, 2017

c. Family functioning variables (diabetes specific burden reported by parents)

d. Increased peer pressure

e. Attempt to gain independence from their parents

f. The effort to discover their self identity

i. Girls pls are at high risk than male.

ii. Depression and other emotional disorders are associated with poor glycemic control in both adolescents and adults resulting in frequent hospital admissions due to episodes of DKA or severe hypoglycemia.

iii. Children with recurrent DKA are more likely to have psychiatric disorders than children in good metabolic control.

iv. Poor glycemic control in adolescents with diabetes and depression may be attributed to a lack of compliance with diet, exercise and medications.

v. In addition, there is an increased incidence of coexisting eating disorders which is associated with intentional insulin omission and thus impaired metabolic control.

vi. Furthermore apathy and lack of self care which are frequently seen in pts with depression or anxiety disorders, can lead to eating or exercise habits that cause diabetic control to deteriorate.

\section{Eating disorders}

A) Disturbed eating behaviours mainly include bulimia nervosa (BN) and binge eating disorder (BED) whereas anorescia nervosa (AN) is rare.

B) More frequently seen in girls than in boys.

C) Insulin omission refers to the skipping or reduction of the prescribed insulin to prevent weight gain and draw the attention of parents mainly observed in $\mathrm{BN}$, also seen in $\mathrm{AN}$ as means of preventing hypoglycemia.

D) Potential risk factors for the development of eating disorders in adolescents. 
a. Female gender, girls between the age of 13-14years

b. Age 13-14years for girls and above 16years for boys

c. Increased body weight (partially attributed to insulin therapy)

d. Constant food preoccupation because of diabetes

e. Presence of eating disorders in parents (ESP the mother)

f. Presence of other psychiatric disorders (such as depression, anxiety or substance abuse)

g. Parental conflicts.

E) An important complication of BN \& BED is poor glycemic control and frequent episodes of DKA. Poor glycemic control is also a complication of AN (due to insulin dose reduction), but a more serious hazard is the frequent hypoglycemic episodes due to skipping meals, which may even be fatal.

F) Eating disorders are also responsible for the increased prevalence of long term diabetic complication, such as retinopathy, neuropathy and nephropathy of poor metabolic control.

\section{Cognitive function and school functioning}

a) Impaired cognitive and learning abilities are more common among boys and in children diagnosed with diabetes during infancy and their preschool years $(<5$ years of age).

b) Diabetic children with early disease onset performed more poorly on measures of intelligence, attention, processing speed and long term memory than control children.

c) Hypoglycemia has been associated with impaired intellectual abilities such as verbal and full scale intelligence scores.

d) Prolonged hyperglycemia has also been associated with cognitive dysfunction.

e) Behavioural disturbances (over activity, lack of concentration, negative mood swings) have been reported in children with hyperglycemia of long duration.

f) Academic achievement and school performance are lower in children with poor metabolic control.

\section{Family functioning}

i. Family factors are integral to the management of diabetes in children.

ii. The family's understanding and involvement as well as support help in the better management.

iii. Family stress and conflict may contribute negatively to the childs diabetic control.

iv. Family support is linked to both short and long term adherence. Family conflict adversely affects adherence and is the strongest predictor of long term adherence.

v. Most adolescents have reported that they expect instrumental support from their families and emotional support from their peers.

\section{Problems economic and social in nature}

i. In addition to these psychological sequelae of type 1 diabetes the family and the pt are also faced with problems economic and social in nature.

ii. Often encountered in lower socio economic group where increasing costs of insulin, blood tests and other medical expenses must be met.

iii. Reduced job opportunities, losing job, shift-work problems.

iv. Withholding information about their condition from their employees.

v. Delay in marriage

Issues concerned with matrimony, which is much more frequent conceding girls rather than boys. These factors must be recognized, repeated counseling of child, parents and family members is necessary. Diabetic educator if necessary should take the guidance of psychologist to visit is family essential for diabetic educator to assess the actual situation. Recognition of counseling of phycho social factors must be constant phenomenon, the diabetes educator.

\section{Adherence/compliance in type I $\mathrm{dm}$}

a. Adherence refers to the extent to which a personic behavior interms of
i. Taking medication
ii. Following diets and exercise
iii. Executing life style changes

b. Non-adherence is a major problem and in general diabetic patients tend to be only partially or inconsistently compliant with their regimen.

c. Adolescents tend to be more non-compliant with their diabetes regimen than children and adults. This has been attributed to a complex interplay of many physiological as well as psychological factors.

d. The period of adolescence appears to be associated with greater non-compliance than the late then years (17-19years).

e. The average age at non-compliance is around 14.8years.

f. Lower levels of compliance were reported during the 1st decade of type $1 \mathrm{DM}$.

g. The 1st episode of non compliance usually begins after 3-4years of diagnosis.

Diabetes educator should familiarize all these fact and also able to recognize the psychosocial aspect and offer proper counseling not only pts and also family members particularly parents of children.

\section{Adolescence}

Psychological problem associated with disregard to follow the diet, taking insulin on time and recolt to the advice by the parents. More common in girls than boys.

\section{Type 2 DM}

More than the psychological factors depression is major problem in T2DM, this may worsen diabetes control and in the long run contribute the complication, economical constrain, diats education should also be able to counsel such pts. 


\section{Acknowledgements}

None.

\section{Conflict of interest}

Author declares that there is no conflict of interest. 